\title{
Is There Still a Place for Revascularisation in the Management of Stable Coronary Artery Disease Following the ISCHEMIA Trial?
}

\author{
Andre Briosa e Gala ${ }^{1}$ and Nick Curzen ${ }^{1,2}$ \\ 1. Coronary Research Group, University Hospital Southampton NHS Foundation Trust, Southampton, UK; \\ 2. Faculty of Medicine, University of Southampton, Southampton, UK
}

DOI: https://doi.org/10.17925/HI.2020.14.1.13

T he ISCHEMIA trial (International study of comparative health effectiveness with medical and invasive approaches; ClinicalTrials.gov Identifier: NCT01471522) has informed practice in patients with stable angina and confirms what other less definitive data have taught us, that in the absence of severe symptoms, significant left main disease or significant left ventricular dysfunction, there is no prognostic benefit of an early invasive/revascularisation strategy with optimal medical therapy (OMT) over OMT alone. Like all quality randomised trials, it has nuances: the invasive treatment group had much better relief of angina than the OMT alone group, and the rate of spontaneous myocardial infarction (MI) in follow-up was lower in the invasive group, although only after a prevalence of periprocedural MI. The clinical outcome consequence of the MI data, if indeed there is one, will only become clear at later follow-up. OMT is a powerful treatment, and reflex revascularisation in patients with little or no angina is not.

\section{Keywords}

ISCHEMIA trial, stable coronary artery disease (CAD), optimal medical therapy (OMT)

Disclosures: Nick Curzen has unrestricted research grants from Boston Scientific, HeartFlow, Haemonetics and Beckmann Coulter; speaker fees/consultancy from Abbott, Boston Scientific, HeartFlow and Haemonetics; and travel sponsorship from Boston Scientific, HeartFlow, Haemonetics,

Edwards and Biosensors. Andre Briosa e Gala has no financial or non-financial relationships or activities to declare in relation to this article.

Review Process: Double-blind peer review.

Compliance with Ethics: This article is an opinion piece and does not report on new clinical data, or any studies with human or animal subjects performed by the authors.

Authorship: The named authors meet the International Committee of Medical Journal Editors (ICMJE) criteria

for authorship for this manuscript, take responsibility for the integrity of the work as a whole and have given final approval for the version to be published.

Access: This article is freely accessible at touchCARDIO.com (c) Touch Medical Media 2020.

Received: 29 May 2020

Accepted: 27 July 2020

Published Online: 8 August 2020

Citation: Heart International. 2020;14(1):13-5

Corresponding Author: Nick Curzen,

Coronary Research Group, University Hospital

Southampton NHS Foundation Trust, University

of Southampton, University Road, Southampton

S017 1BJ, UK. E: nick.curzen@uhs.nhs.uk

Support: No funding was received in

the publication of this article.
Optimal medical therapy (OMT) is the cornerstone of management in patients with coronary atheroma, given the substantial evidence that it can delay disease progression and damp down both chronic and acute vascular inflammation, thereby leading to a reduction in cardiovascular events and mortality. ${ }^{12}$ In this context, revascularisation, with either percutaneous coronary intervention (PCI) or coronary artery bypass graft (CABG), can be viewed as an adjunct to OMT. ${ }^{1}$ The exact nature of the role of revascularisation as an adjunctive treatment is dependent upon a number of factors, perhaps the most important of which is the mode of clinical presentation. specifically, the evidence demonstrating the outcome benefit of coronary revascularisation in the context of both ST-elevation myocardial infarction (STEMI) and non-ST-elevation myocardial infarction (NSTEMI) is overwhelming. By contrast, the additional benefit of revascularisation above and beyond OMT in patients with stable angina has been much harder to demonstrate with consistency. The reasons for this relate to the heterogeneity of the studies and trials that provide resources to this evidence base. For example, randomised trials comparing pressure wire versus angiogram alone-directed $\mathrm{PCl}$ indicate a consistent benefit in terms of clinical outcome. ${ }^{3-7}$ However, greater scrutiny is required to understand: (a) that these patient populations had already had diagnostic angiography and been triaged towards PCI before the pressure wire was utilised to assess vessel-specific ischaemia, and (b) that the nature of the outcome benefit does not include reduction in mortality, nor, in most cases, in myocardial infarction (MI). By contrast, observational data indicate that revascularisation is effective at reducing angina and, under some circumstances, improving prognosis (especially by meta-analysis), particularly in those with high-risk anatomy (left main stenosis $>50 \%$ ), multivessel disease or severely impaired left ventricular function. ${ }^{8}$

The waters of our understanding are muddied by other data suggesting that the presence and extent of ischaemia itself is associated with higher rates of $\mathrm{Ml}$ and cardiovascular death.? The enticing and plausible notion that revascularisation should therefore be associated with a consequent reduction in risk of $\mathrm{Ml}$ and death in stable patients has, however, been difficult to substantiate. Until recently, the Clinical Outcomes Utilizing Revascularisation and Aggressive DruG Evaluation trial (COURAGE; NCT00007657) stood out as the most definitive attempt to address this question..$^{10}$ The headline result was that PCI plus OMT, over and above OMT alone, did not reduce death or major cardiovascular events in patients with significant coronary artery disease. ${ }^{11}$ There were, inevitably, a number of perceived limitations of the trial design and its interpretation, summarised here. ${ }^{12}$ The true clinical value of OMT targeted to patients who have been identified as having coronary atheroma by non-invasive computed tomography coronary angiogram (CTCA) is demonstrated by the significant reduction in hard clinical endpoints at 5 years seen in the CTCA group in the randomised Scottish COmputed Tomography of the HEART trial (SCOT HEART; NCT01149590). .13,14 $^{2}$ 


\section{The trial - headline results}

Given ongoing uncertainties and discrepant data, the recently presented International Study of Comparative Health Effectiveness with Medical and Invasive Approaches (ISCHEMIA; NCT01471522) trial was designed to answer the essential question: whether there is a prognostic benefit of additional cardiac catheterisation and, where feasible, revascularisation ( $\mathrm{PCl}$ or $\mathrm{CABG}$ ) to $\mathrm{OMT}$ in patients with stable angina and at least moderate ischaemia on non-invasive stress testing. ${ }^{15,16}$ The primary endpoint was a composite outcome that included cardiovascular death, MI, hospitalisation for unstable angina, heart failure or resuscitated cardiac arrest. Key secondary endpoints included time to death or MI, angina status and quality of life.

Approximately $40 \%$ of the 8,518 patients screened were excluded due to lack of significant ischaemia on stress testing or left main stem stenosis of at least $50 \%$ or non-obstructive coronary artery disease on CTCA. Of the 5,179 patients randomised, 2,588 patients were assigned to an invasive strategy (OMT and revascularisation) and 2,591 patients to a conservative strategy (OMT). Over a median follow-up of 3.2 years, there was no significant difference in the rates of the combined primary endpoint (13.3\% in the invasive strategy and $15.5 \%$ in the conservative strategy [hazard ratio, HR=0.93, 95\% confidence interval 0.80-1.08]). Moreover, rates of 'hard' secondary endpoints such as all-cause death, cardiovascular death and MI were similar in both treatment arms. Of note, all-cause death rate was low in both groups $(6.4 \%$ in the conservative strategy and $6.5 \%$ in the invasive strategy $[p=0.67])$, which highlights the efficacy of guideline-directed OMT. The headline result from ISCHEMIA was therefore, similarly to COURAGE over a decade before, that an invasive strategy plus OMT yielded no prognostic benefit above and beyond OMT alone in patients with stable angina.

\section{Interpretation - forensic assessment with an interventional bias}

ISCHEMIA has produced an important and timely result, that should modify any tendency for reflex coronary angiography and revascularisation for patients who present with stable angina before they are established on OMT and should prevent such patients acquiring the impression that revascularisation has prognostic benefit when they are considering their management options. However, in common with all trials, some aspects of ISCHEMIA warrant forensic scrutiny in order to interpret the results in the context of the patients we look after.

Firstly, although the inclusion criteria were broad and well defined, some of the exclusion criteria removed patients in whom revascularisation might be expected to be of greatest benefit. Specifically, patients with severe angina (Canadian Cardiovascular Society [CCS] grade III-IV), those with left main stem stenosis of $50 \%$ or more, and left ventricular ejection fraction $<35 \%$ were excluded. It is unclear why these groups were excluded, particularly those with class III angina. Certainly, by excluding these subgroups, our ability to translate the ISCHEMIA trial results into our real-life 'all comers' practice is more challenging.

Secondly, despite the intention to recruit patients with angina and at least moderate reversible myocardial ischaemia, $12 \%$ of the study population had only mild ischaemia, and just over one-third had no angina at baseline. Enrolling patients with milder degrees of ischaemia potentially reduces the power to detect a difference between the groups, and therefore any benefit of revascularisation. In terms of a potential difference between the strategies, this potentially compounds the failure to include patients in the trial who had severe angina.
Thirdly, and with regard to the primary endpoint, the lack of difference after a median follow-up of 3.2 years is unequivocal. The same is true of cardiovascular death, in isolation. The breakdown analysis of $\mathrm{Ml}$ is, however, more complex. Unsurprisingly, patients who underwent revascularisation had a higher rate of periprocedural Ml. The outcome implications of periprocedural $\mathrm{Ml}$ are unclear in all but the most clinically obvious MI. If this was not the case, then a routine post-PCl check of cardiac enzymes would be mandatory, whereas, in fact, they are rarely undertaken in the UK. By contrast, the rate of spontaneous $\mathrm{Ml}$ after the periprocedural time frame shows a divergence over the remaining follow-up period that favours the invasive group. Spontaneous MI is generally considered to be associated with long-term outcome, and so it remains to be seen whether this difference is clinically relevant in longer follow-up. Given the divergence in the event curves in the trial as reported so far, it will be interesting to track longer-term outcomes to see if this trend continues; the value of such a longer-term follow-up strategy was recently seen in the SYNTAX study: TAXUS drug-eluting stent versus coronary artery bypass surgery for the treatment of narrowed arteries trial (NCT00114972). ${ }^{17,18}$

Fourthly, just under one quarter of the patients in the OMT arm underwent coronary revascularisation by the end of the study. This is surely an important statistic when it comes to counselling patients. Despite having already excluded from the trial those patients who had more severe angina at the start (CCS grade III-IV), symptoms dictated that, despite OMT, nearly one in four would have to undergo revascularisation. This is a slightly lower figure to that seen in the OMT group in COURAGE.

Finally, the separate presentation and paper regarding the quality-of-life outcomes in ISCHEMIA yield further extremely important information to furnish the decision-making processes for the patient and their family. ${ }^{18}$ Specifically, for those patients in the trial who actually had angina at baseline, the relief of symptoms was vastly superior in the invasive cohort. Since angina was most likely the driver for the patient to be seeing a cardiologist in the first place, relief of the symptom seems likely to be deemed of considerable value to them.

The results of the ISCHEMIA trial are consistent with those of COURAGE. Like in COURAGE, they can be summarised as follows: there is no major prognostic advantage of adding an invasive strategy to OMT in patients with stable angina, but revascularisation is associated with a higher chance of being rendered angina-free, without any overall cost in terms of adverse outcomes.

\section{Speculation - the ISCHEMIA trial in context}

From an interventional cardiologist's point of view, what could have affected the results of such a trial? There is, firstly, a circle to square: how is it possible that with the weight of evidence from established randomised trials that show an outcome benefit from using pressure wire-directed $\mathrm{PCl}$ versus angiogram-directed (DEFER, FAME [NCT00267774], FAME 2 [NCT01132495], DEFINE FLAIR [NCT02053038], iFR SWEDEHEART [NCT02166736]), that there is no obvious outcome

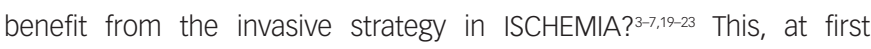
glance, seems like a very large elephant in the room. It is tempting to speculate that a mandated pressure wire-directed vessel-specific revascularisation strategy, as part of ISCHEMIA, may have produced even better results for that arm, despite fewer stents and possibly less $\mathrm{CABG}$, with correspondingly less periprocedural MI. However, regardless of this speculation, ISCHEMIA should be taken as a warning to the over-enthusiastic interventionalist. It should act as a reminder that 
in none of the pressure-wire trials mentioned above was there any mortality benefit. So, we need to ensure that our patients are equipped with all this information.

A second important question addresses how ISCHEMIA will fit into modern clinical practice. Of course, in general, patients were risk stratified in a two-stage approach: a functional test to identify ischaemia, and an anatomical test to confirm the presence and extent of obstructive coronary artery disease. This two-step assessment may already be inefficient and suboptimal in modern healthcare, and soon, it may become obsolete in many cases. Adopting the ISCHEMIA protocol in routine clinical practice is unlikely to be feasible due to high healthcare costs and significant delays in scheduling two separate tests. Recent technological advancements in the field of CT angiography now permit a simultaneous anatomical and functional non-invasive assessment of epicardial coronary vessels in the form of CT-derived fractional flow reserve (FFRCT), thus providing a more elegant diagnostic pathway. The validation and clinical utility of FFRCT are already established and reviewed elsewhere. ${ }^{24}$ The FORECAST trial (Fractional flow reserve derived from computed tomography coronary angiography in the assessment and management of stable chest pain; NCT03187639) is a multicentre, randomised controlled trial comparing routine FFRCT to routine care in 1,400 patients presenting with stable chest pain. ${ }^{25,26}$ The results of FORECAST will be presented later this year, and may inform the widespread introduction of FFRCT as a default primary assessment test for patients with stable chest pain. The beauty of this approach in the context of ISCHEMIA is that patients will get a definitive non-invasive diagnosis regarding presence and extent of atheroma and ischaemia, all of which can then be treated with OMT alone, unless symptoms dictate that an invasive approach would be helpful.

\section{Conclusions \\ What lessons are we to learn from the ISCHEMIA trial?}

First, results are consistent with previous trials, such as COURAGE, and reinforce the role of OMT as a first-line therapy. ${ }^{1.11}$ Second, in the absence of left main stem stenosis, left ventricular ejection fraction $<35 \%$ and highly symptomatic angina (CCS grade III-IV), there is no prognostic benefit of revascularisation above and beyond OMT. Third, the strategy of OMT and revascularisation is significantly more likely to provide symptomatic relief in patients with angina on OMT. Fourth, that longer-term follow-up is required to see if the lower rate of spontaneous $\mathrm{MI}$ in the invasive arm in ISCHEMIA is of prognostic relevance.

What impact will the ISCHEMIA trial have? It should confirm what we already knew, that there is no mandate to perform revascularisation in patients with stable angina who are on OMT and whose symptoms are acceptable once on this therapy. It should also confirm that revascularisation will be an excellent adjunctive therapy in such patients if their angina is affecting the quality of their lives.
1. Knuuti J, Wijns W, Saraste A, et al. 2019 ESC Guidelines for the diagnosis and management of chronic coronary syndromes. diagnosis and management of

2. Baigent $C$, Keech A, Kearney PM, et al. Efficacy and safety of cholesterol-lowering treatment: prospective meta-analysis of data from 90,056 participants in 14 randomised trials of statins. Lancet. 2005;366:1267-78.

3. Bech GJ, De Bruyne B, Pijls NH, et al. Fractional flow reserve to determine the appropriateness of angioplasty in moderate coronary stenosis: a randomized trial. Circulation. 2001;103:2928-34.

4. Tonino PAL, De Bruyne B, Pijls NH, et al. Fractional flow reserve versus angiography for guiding percutaneous coronary intervention. N Eng/ J Med. 2009;360:213-24.

5. De Bruyne B, Pijls NH, Kalesan B, et al. Fractional flow reserve-guided PCI versus medical therapy in stable coronary reserve-guided PCl versus medical therapy in

6. Davies JE, Sen S, Dehbi H-M, et al. Use of the instantaneous wave-free ratio or fractional flow reserve in PCI. N Eng/ I Med. 2017;376:1824-34

7. Neumann FJ, Sousa-Uva M, Ahlsson A, et al. [2018 ESC/EACTS Guidelines on myocardial revascularization]. Kardiol Pol. 2018;76:1585-664

8. Iskander S, Iskandrian AE. Risk assessment using single-photon emission computed tomographic technetium-99m sestamibi imaging. J Am Coll Cardiol. 1998;32:57-62.

9. Boden WE, O'Rourke RA, Teo KK, et al. Optimal medical therapy with or without PCI for stable coronary disease. N Eng/ J Med. 2007;356:1503-16.

10. ClinicalTrials.gov. Clinical outcomes utilizing revascularization and aggressive drug evaluation. Available at: www.clinicaltrials.
gov/ct2/show/NCT00007657 (accessed 5 August 2020).

11. Curzen NP. Is there evidence for prognostic benefit following $\mathrm{PCl}$ in stable patients? COURAGE and its implications: an interventionalist's view. Heart. 2010;96:103-5.

12. Newby $D E$, Adamson PD, Berry C, et al. Coronary C angiography and 5 -year risk of myocardial infarction. N Engl J Med. 2018;379:924-33.

13. Maron DJ, Hochman JS, Reynolds HR, et al. Initial invasive or conservative strategy for stable coronary disease. N Engl I Med. 2020;382:1395-407.

14. ClinicalTrials.gov. Scottish computed tomography of the heart trial (SCOT-HEART). Available at: www.clinicaltrials.gov/ct2/ show/NCT01149590 (accessed 5 August 2020).

15. ClinicalTrials.gov. International study of comparative health effectiveness with medical and invasive approaches (ISCHEMIA). Available at: https:///linicaltrials.gov/ct2/show/ (ISCHEMIA). Available at: https://clinicalt)

16. Thuijs DJFM, Kappetein AP, Serruys PW, et al. Percutaneous coronary intervention versus coronary artery bypass grafting in patients with three-vessel or left main coronary artery disease: 10-year follow-up of the multicentre randomised controlled SYNTAX trial. Lancet. 2019;394:1325-34.

17. Spertus JA, Jones PG, Maron DJ, et al. Health-status outcomes with invasive or conservative care in coronary disease. N Engl J Med. 2020;382:1408-19.

18. ClinicalTrials.gov. SYNTAX study: TAXUS drug-eluting stent versus coronary artery bypass surgery for the treatment of narrowed arteries (SYNTAX). Available at: www.clinicaltrials. gov/ct2/show/NCT00114972 (accessed 5 August 2020)

19. Götherg M, Christiansen EH, Gudmundsdottir Instantaneous wave-free ratio versus fractional flow reserve to guide PCl. N Eng/ / Med 2017:376:1813-23.

20. ClinicalTrials.gov. Evaluation of iFR VS FFR in stable angina or acute coronary syndrome (iFR Swedeheart). Available at: https://clinicaltrials.gov/ct2/show/NCT02166736 (accessed 5 August 2020).

21. ClinicalTrials.gov. Fractional flow reserve versus angiography for multivessel evaluation (F.A.M.E.). Available at: www.clinicaltrials. gov/ct2/show/NCT00267774 (accessed 5 August 2020).

22. ClinicalTrials.gov. FAME II - Fractional flow reserve (FFR) guided percutaneous coronary intervention ( $\mathrm{PCl}$ ) plus optimal medical treatment (OMT) verses OMT. Available at: www.clinicaltrials. gov/ct2/show/NCT01132495 (accessed 5 August 2020).

23. ClinicalTrials.gov. Functional lesion assessment of intermediate stenosis to guide revascularisation (DEFINE-FLAIR). Available at: https://clinicaltrials.gov/ct2/show/NCT02053038 (accessed 5 August 2020).

24. Rajani R, Modi B, Ntalas I, Curzen N. Non-invasive fractional flow reserve using computed tomographic angiography: where are we now and where are we going? Heart. 2017;103:1216-22.

25. ClinicalTrials.gov. Fractional flow reserve derived from computed tomography coronary angiography in the assessment and management of stable chest pain (FORECAST). Available at: https://clinicaltrials.gov/ct2/show/NCT03187639 (accessed 5 August 2020)

26. Mahmoudi M, Nicholas Z, Nuttall J, et al. Fractional flow reserve derived from computed tomography coronary angiography in the assessment and management of stable chest pain: Rationale and design of the FORECAST trial. Cardiovasc Revasc Med. 2019. doi: 10.1016/j.carrev.2019.12.009 [Online ahead of print]. 